\title{
The effect on radioresistance of manganese superoxide dismutase in nasopharyngeal carcinoma
}

\author{
YAMING QU ${ }^{1}$, HUA ZHANG $^{1}$, SUPING ZHAO ${ }^{1}$, JIDONG HONG ${ }^{2}$ and CANE TANG $^{3}$ \\ Departments of ${ }^{1}$ Otolaryngology Head and Neck Surgery and ${ }^{2}$ Radiation Oncology, and ${ }^{3}$ Central Laboratory of \\ Medical Research, Xiang Ya Hospital, Central South University, Xiangya Road 87, 410008 Changsha, P.R. China
}

Received November 8, 2009; Accepted December 30, 2009

DOI: 10.3892/or_00000726

\begin{abstract}
Failure to control nasopharyngeal carcinomas (NPC) is mainly due to a portion of radioresistant phenotype. Identifying gene targets for radiosensitization is an important strategy in improving anticancer treatments. Exposure of cells to ionizing radiation leads to the formation of reactive oxygen species that are associated with radiation-induced cellular apoptosis and necrosis. The antioxidant enzyme manganese superoxide dismutase (SOD2) catalyzes the dismutation of the superoxide anions into hydrogen peroxide. We reasoned that SOD2 could contribute to the radioresistant phenotype in NPC cells. We compared CNE1 cells with CNE2 cells in radiation character and SOD2 protein. SOD2 gene silencing through the plasmid transfer using a microRNA interference optimized for transcription in NPC cell lines inhibited the radioresistance of human NPC cells. We compared radioresistant NPC with radiosensitive NPC in SOD2 expression. CNE1 cells and CNE2 cells demonstrated dose-modifying factors at $10 \%$ isosurvival of 1.529 and 1 . CNE1 cells were 1.94-fold higher than CNE2 cells at SOD2 protein baseline, and CNE1 cells exposed to ionizing radiation demonstrated 1.15- to 1.39-fold increase in SOD2 immunoreactive protein. Radioresistance in CNE1 cells was reduced following expression of miRNA targeting SOD2. Radioresistant tumors (11/23) were SOD2-positive, and 2/46 radiosensitive tumors were SOD2-positive before commencement of radiotherapy. The results presented suggest that SOD2 expression can participate in radioresistance of NPC, being markers of a subset of tumors in which routine radiation treatment failure is likely. Combination of the SOD2 gene silencing therapy and conventional radiotherapy should be attempted to improved cancer therapy for NPC.
\end{abstract}

Correspondence to: Dr Suping Zhao, Department of Otolaryngology Head and Neck Surgery, Xiang Ya Hospital, Central South University, Xiangya Road 87, 410008 Changsha, P.R. China E-mail: nantian9@126.com

Key words: manganese superoxide dismutase, nasopharyngeal carcinoma, radioresistance

\section{Introduction}

The intrinsic radioresistance of the cells is among the possible reasons for the failure of radiation treatment to control the tumor. Improving the therapeutic ratio thus requires knowledge of the radiobiological factor that could influence tumor control by fractionated radiation treatment. Exposure of eukaryotic cells to ionizing radiation (IR) results in the formation of free radicals and in oxidative damage to biomolecules such as DNA, proteins and lipids that contribute to the biological effects of radiation (1-3). Manganese superoxide dismutase (MnSOD now known as SOD2) is a mitochondrial enzyme that catalyzes the dismutation of superoxide anion $\left(\mathrm{O}_{2}{ }^{-}\right)$to form hydrogen peroxide $\left(\mathrm{H}_{2} \mathrm{O}_{2}\right)$ and molecular oxygen $\left(\mathrm{O}_{2}\right)$ (4). SOD2 works in concert with other antioxidant enzymes to defend cells against the damage associated with exposure to reactive oxygen species (ROS) (5) and is thought to be an important determinant of sensitivity to ROS-induced cytotoxicity. Scavenging the reactive species produced at the time of irradiation can mitigate some of the effects of IR-iduced injury. Active SOD enzymes and SOD mimetic compounds can lead to inhibition of the deleterious effects induced by IR in a wide variety of in vitro and in vivo studies ranging from transformation assays, bystander effects, inflammatory responses and fibrosis (6-12). The protective effect of SOD2 against normal tissue damage caused by radiation is highlighted by in vivo experiments showing induction of SOD2 following radiation in the heart and gut $(13,14)$ and the finding that overexpression of SOD2 in normal mouse epithelial tissues protected them from radiation injury (15).

Despite expression of endogenous SOD2 has been found to be reduced in many human cancer cells and transformed cell lines (16-18). Expression of SOD2 causes significant alterations in the malignant phenotype as well as inhibition of tumor growth in vivo $(16,18,19)$. In many human tumor cells, SOD2 immunoreactive protein and activity are barely detectable in contrast to other AEs (CuZnSOD, CAT and GPx) (20). Therefore, increase of endogenous SOD2 could contribute to radioresistance seen in some tumor cells treated with radiation. A fraction of genes in the radiation-induced gene expression profiles in tumor cells represent genes that are regulated by SOD2 induction (8). Identifying SOD2 gene could therefore yield crucial insights into the genes in radioresistant phenotypes. 
Few studies have been undertaken to assess whether expression of SOD2 can be involved in the radioresistance of cancers and the results of these studies are contradictory $(15,21-23)$. Yamaguchi et al found that endogenous SOD activity protects human leukemic and cancer cells from radiation. Veldwijk et al reported that the human superoxidedismutase gene does not confer radioresistance on HeLa cervical carcinoma cells.

To determine in the present study if a causal relationship between SOD2 expression and radioresistance exists in human nasopharyngeal carcinoma (NPC) cells, gene expression of SOD2 in CNE1 cells was compared to that in CNE2 cells. Increased expression of SOD2 protein was detected in radioresistant CNE1 cells, relative to that in CNE2. The involvement of SOD2 in the IR-induced alterations in gene expression was confirmed by a lack of radiation responsiveness in radiosensitive CNE2 cells. Moreover, expression of miRNA for SOD2 inhibited the up-regulation of SOD2 in CNE1 cells, as well as inhibiting the radiation-resistant phenotype. Finally, SOD2 expression using immunohistochemical staining on paraffin tissue sections was associated with radioresistant NPC. These results provide strong evidence that SOD2 expression induces the radioresistant phenotype of human nasopharyngeal carcinoma following radiation exposure.

\section{Materials and methods}

Cell cultures. Human nasopharyngeal carcinoma cell lines CNE1 snd CNE2 were purchased from Xiang Ya Central Experiment Laboratory, Central South University and utilized because relatively characterized radioresistance among NPC cell lines was available in these cell lines. Cells were grown in Rosswell Park Memorial Institute 1640 medium (RPMI1640, Invitrogen, Carlsbad, CA, USA) supplemented with $10 \%$ fetal bovine serum (FBS, Longgreen Co., China) and non-essential amino acids. Cells were maintained at $37^{\circ} \mathrm{C}$ and $5 \% \mathrm{CO}_{2}$ during all experiments.

Generation of miRNA expression vectors. The pcDNATM6.2GW/EmGFP-miR Vectors were purchased from Invitrogen Co. The vectors are based on knockdown cassettes driven by RNA polymerase II promoters that allow expression of engineered miRNA sequences and endogenous murine miR-155 flanking sequences that allow proper processing of the miRNA, which permits visual detection of cells expressing the miRNA through co-cistronic expression of EmGFP. The pcDNATM6.2-GW/EmGFP-miR-SOD2 plasmid was constructed by inserting a double-stranded oligonucleotide (ds oligo) encoding a pre-miRNA for SOD2 into pcDNATM6.2GW/EmGFP-miR. The ds oligo insert was synthesized by Invitrogen Corp. (Shanghai, China) and cloned into the site adjacent miR-155 flanking region of expression cassette. The pre-miRNA ds oligo relating to SOD2 utilized in this study is as follows: top strand, 5'-TGCTGTAAGCGTGCTCCCAC ACATCAGTTTTGGCCACTGACTGACTGATGTGTGAG CACGCTTA-3'; bottom strand, 5'-CCTGTAAGCGTGCTC ACACATCAGTCAGTCAGTGGCCAAAACTGATGTGTG GGAGCACGCTTAC-3' The construct was transformed into competent E.coli that was in cultured in the selecting medium with $50 \mu \mathrm{g}$ of spectinomycin/ml. Expression construct of the selected plasmid was confirmed by DNA sequencing. The pcDNATM6.2-GW/EmGFP-miR-neg control plasmid (Invitrogen Co.) contains an insert (the sequence without 5' overhangs: 5'-GAAATGTACTGCGCGTGGAGACGTT TTGGCCACTGACGACGTCTCCACGCAGTACATTT-3') that can form a hairpin structure that is processed into mature miRNA, but is predicted not to target any known vertebrate gene.

Establishment of cell lines that expressed miRNA SOD2. CNE1was transfected with plasmid encoding the miRNA expression vectors described above using the cationic lipidbased Lipofectamine ${ }^{\mathrm{TM}} 2000$ (Invitrogen Co.). Two days after transfection, cells harboring an expressing integrant were viewed by fluorescence microscopy based on GFP. The cells were transfected for $72 \mathrm{~h}$, trypsinized, and cultured in the selecting medium with $4 \mu \mathrm{g}$ of blasticidin/ml for 14 days. The transfected cells were cultured for at least two passages in blasticidin-free medium before the experiments.

Clonogenic survival after irradiation. Radioresistance was measured by clonogenic survival following exposure to IR. Control and pcDNA ${ }^{\mathrm{TM}}$ 6.2-GW/EmGFP-miR-neg control plasmid and pcDNA ${ }^{\mathrm{TM}}$ 6.2-GW/EmGFP-miR-SOD2 expression construct infected cells were trypsinized and plated in triplicate at cell population of 100, 100, 200, 400, 10 $30^{3}, 10^{4}$ and $10^{5}$ per $3 \mathrm{~cm}^{2}$ dish respectively, then were irradiated with 0-8 Gy using medical linear accelerator. Irradiated cells were trypsinized and cloned in a $37^{\circ} \mathrm{C}$ incubator with $5 \% \mathrm{CO}_{2}$ for 14 days. Clones containing more than 50 cells were scored as survivors, and the data were normalized to the appropriate sham-irradiated control group. Dose-modifying factors (DMFs) were calculated using the following expression: $\mathrm{DMF}=($ dose to reach the specified survival in resistant cells $) /$ (dose to reach the same survival in the control). A linear quadratic equation was fit to the radiation dose response data using the programme DRFIT (24). The parameters obtained from the fitted data were $\alpha$ (a measure of the initial slope), $\beta$ (a measure of the final slope), MID (mean inactivation dose, the area under the curve in linear coordinates), and SF2 (surviving fraction at $2 \mathrm{~Gy}$ ).

Immunoblotting techniques. Wild-type CNE1, CNE1 ${ }^{+}$miR-neg and $\mathrm{CNE}^{+}{ }^{+}$miR-SOD2 cells were cultured in $10 \%$ FBSRMPI-1640, and total protein extracts were prepared. Equal quantities of sample protein were mixed with $40 \mu 1$ of loading buffer, heated at $95^{\circ} \mathrm{C}$ for $10 \mathrm{~min}$, size separated in $12 \%$ acrylamide SDS-polyacrylamide gel electrophoresis, and transferred to polyvinylidene fluoride membrane (PVDF, Millipore Co. USA). The membrances were then blocked at room tempe-rature for $2 \mathrm{~h}$ in $5 \%$ non-fat dry milk solution, washed with $0.01 \%$ Tween-phosophate-buffered saline, and incubated overnight at $4{ }^{\circ} \mathrm{C}$ with either of the following primary antibodies: goat anti-SOD2 at a dilution of 1:500; anti- $\beta$-actin at a dilution of 1:400 (Santa Cruz Biotechnology, Santa Cruz, USA). The blots were then incubated with horseradish peroxidase-conjugated secondary antibody at a dilution of 1:500. Protein bands were visualized using the DAB detection kit (Maixin Biotechnology, Fuzhou, China). 

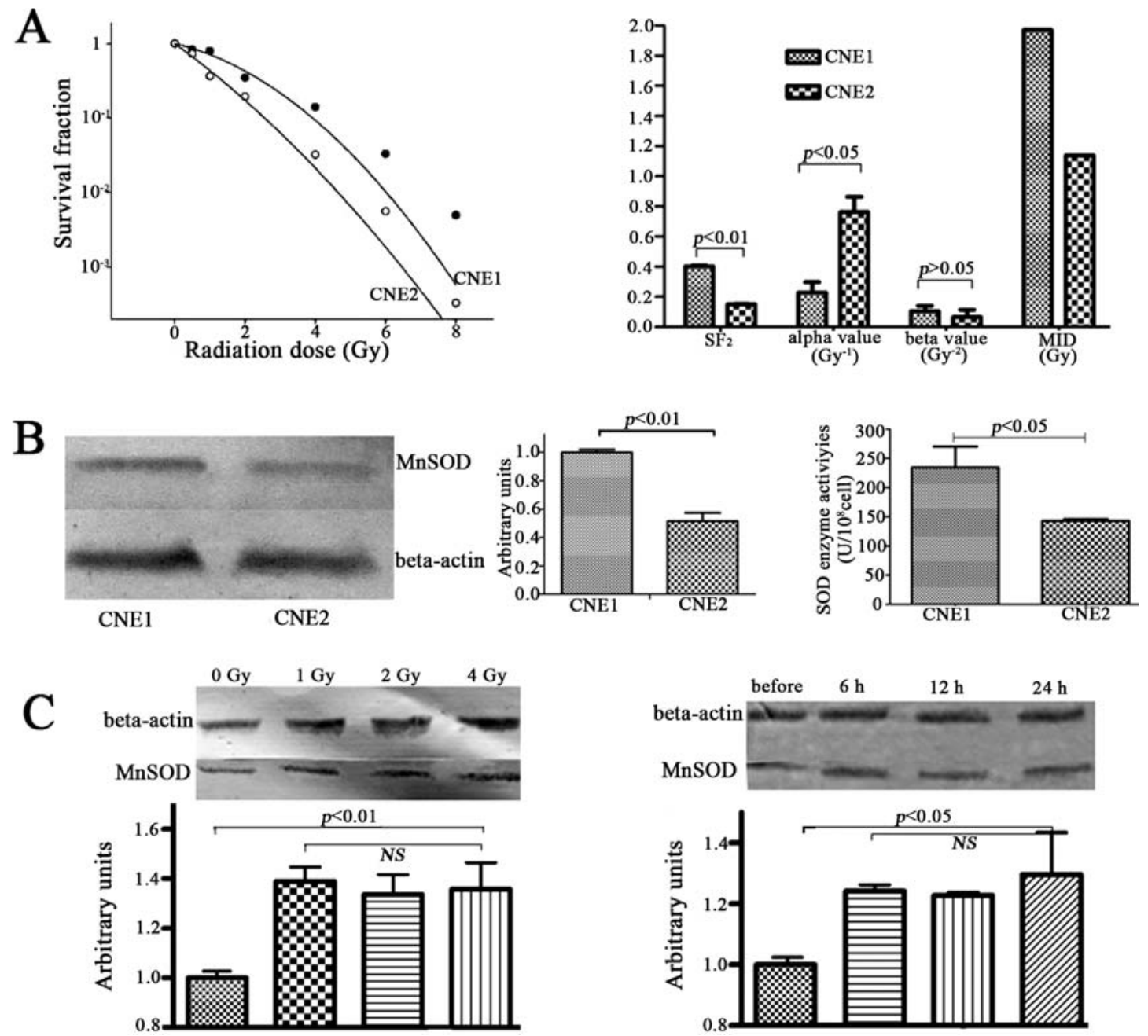

Figure 1. Radioresistance and SOD2 expression. (A) Radioresistance of NPC cell lines. Clonogenic survival of CNE1 and CNE2 cells was measured following exposure to X-irradiation. Plating efficiencies for CNE1 and CNE2 cells were 0.9 and 0.92 , respectively. Results were normalized to non-irradiated cells [data represent mean \pm 1 standard deviation (SD) of two independently irradiated cultures, each plated into three cloning dishes that were counted]. (B) Increased SOD2 expression in CNE1 cells. Immunoreactive protein levels were detected by Western blotting using goat antibody to human SOD2 and B-actin as the loading control. In all cases, the quantitation was done using densitometry and presented as arbitrary units after normalization to $\beta$-actin (three experiments were done in each case and one representative image is shown; the errors represent \pm 1 standard deviation of three separate densitometric analyses done on the single representative image). (C) IR-induced SOD2 expression in CNE1 cells but not in CNE2 cells. Left image, CNE1 cells were irradiated at room temperature with doses of 1,2 and $4 \mathrm{~Gy}$, respectively and harvested using trypsin $24 \mathrm{~h}$ after radiation or sham treatment. Right image, CNE1 cells were irradiated with a single dose of $2 \mathrm{~Gy}$ and harvested using trypsin 6, 12 and $24 \mathrm{~h}$ after radiation or sham radiation. Immunoreactive SOD2 protein levels were analyzed by immunoblotting. Aliquots of above protein ( $25 \mu \mathrm{g} / \mathrm{lane})$ were separated using SDS-12\% polyacrylamide gel electrophoresis and transferred to membranes, and membranes were incubated with antibody and visualized using a DAB detection system with $B$-actin as the loading control.

Reverse transcription-PCR (RT-PCR). Total RNA was isolated using TRIzol reagent (Invitrogen Co.). After confirmation of the RNA integrity on an agarose gel, total RNA was digested using RNase-free DNase I for 20 min, extracted with phenolchloroform, then precipitated with 2.5 volumes of ethanol. The cDNA was synthesized from $2 \mu \mathrm{g}$ of total RNA using RevertAid ${ }^{\mathrm{TM}} \mathrm{M}-\mathrm{MuLV}$ reverse transcriptase and oligo(dT) (MBI Fermentas Life Sciences). For PCR, $5 \mu 1$ of cDNA products was mixed with PCR buffer (Promega, Madison, $\mathrm{WI}$ ) and $0.1 \mu \mathrm{M}$ concentrations of human SOD2 PCR primers synthesized according to the sequence in GenBank. The primers sequences utilized in this study are as follows: SOD2 forward, 5'-CGACCTGCCCTACGACTA-3'; SOD2 reverse, 5'-ACCTGAGCCTTGGACACC-3' and glyceraldehydes-3phosphate dehydrogenase (GAPDH) forward, 5'-CAAGGTC ATCCATGACAACTTTG-3'; GAPDH reverse, 5'-CAAGGT
CATCCATGACAACTTTG-3'. Gene expression levels were estimated by densitometry.

Patients and immunohistochemical staining. Sixty-nine patients were studied from Department of Radiation Oncology, Xiang Ya Hospital between January 2005 and October 2006. These patients were aged $\leq 78$ years, had histologically confirmed poorly-differentiated squamous cell in nasopharynx which was UICC stage II or III, and had not been previously treated. All patients were treated with 70 Gy using medical linear accelerator and were followed up $>6$ months. Formalin-fixed, paraffin-embedded tumor tissue was analyzed from 23 patients (18 males and 5 females) with uncontrolled local or regional tumor and 46 patients (36 males and 10 females) with well-controlled tumor. Immunohistochemical staining of SOD2 was performed on paraffin 

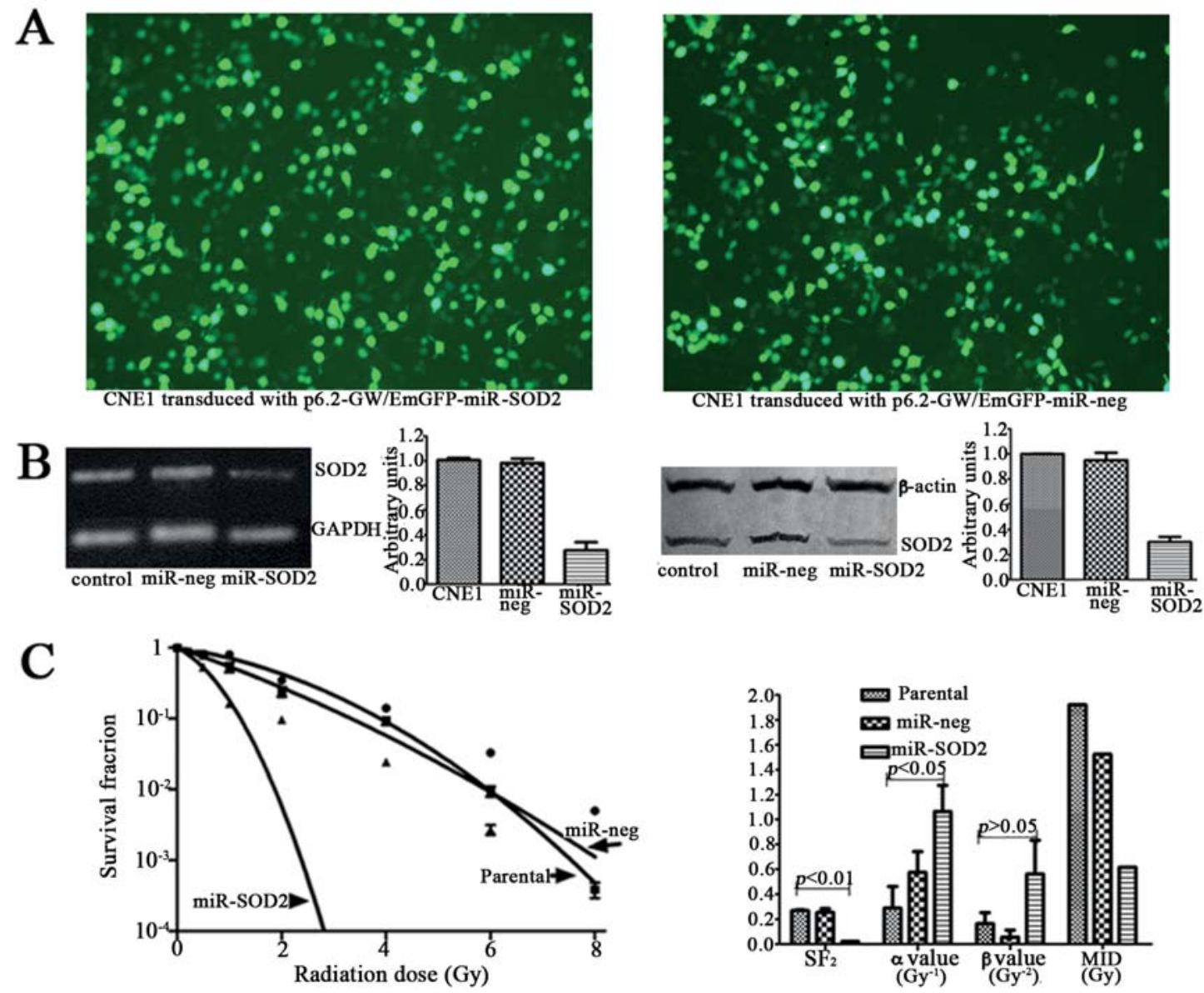

Figure 2. Inhibition of SOD2 expression sensitizes CNE1 cells to radiation. (A) Fluorescence activated cells of the CNE1 cell lines transduced with the miRNA expression vectors. (B) Inhibition of SOD2 expression in CNE1 cells stably transfected with pcDNATM6.2-GW/EmGFP-miR-SOD2 expression construct, compare to uninduced CNE1 cells and CNE1 cells stably transfected with pcDNATM6.2-GW/EmGFP-miR-neg control plasmid. SOD2 expression was measured by RT-PCR using $5 \mu \mathrm{g}$ of total RNA. PCR fragments were enhanced for 30 cycles, and relative levels of SOD2 transcripts were estimated by densitometric analysis. Primers for GAPDH were included as internal controls (three experiments were performed, and a single representative analysis is shown). Densitometry analysis is shown in arbitrary units normalized to the GAPDH loading control (errors represent \pm 1 SD of three separate densitometric analyses done on the single representative set of samples shown above). Immunoreactive SOD2 protein was monitored in uninduced CNE1 controls, CNE1 transduced with miR-neg control plasmid, CNE1 transduced with miR-SOD2 expression vectors. ß-actin was included as the loading control, and the experiment was done three times with one representative blot. Expression levels were measured using densitometry and normalized to the levels seen in the uninduced CNE1 cells (left panel, the data represent the mean \pm 1 standard deviation of three densitometric analyses of the single blot which is shown). (C) Radioresistance was decreased in CNE1 cells transduced with miR-SOD2 expression construct. Parental CNE1 and CNE1 transduced with miR-neg control plasmid were exposed to range of IR doses, and clonogenic survival was determined. The surviving fraction was normalized to non-irradiated cells from each group (data represent mean \pm 1 standard deviation of three separate experiment), the irradiated group, CNE1 with miR-SOD2, is significantly different from the irradiated control, CNE1 with miR-neg, at the 8-Gy dose (paired Student's t test, $\mathrm{p}<0.01$ ).

sections using the streptavidin-biotin peroxidase method. Briefly, 6-mm sections were dewaxed and endogenous peroxidase was blocked by $20 \mathrm{~min}$ pretreatment with $0.5 \%$ $\mathrm{H}_{2} \mathrm{O}_{2}$ in $70 \%$ methanol. Following rehydration, non-immune goat serum (1:30) was used to block non-specific staining. The primary antibody used for SOD2 detection was BMS122 (Bender Co., Austria; dilution 1:100). Tissues were then incubated with biotinylated anti-rabbit $\operatorname{IgG}$ and avidin-biotin peroxidase complex (Maixin Biotechnology) in sequence, with $0.05 \%$ diaminobenzidine as substrate. Sections were lightly counterstained with Harris haematoxylin. Controls were a known positive specimen from a nasopharyngeal mucous membrane expressing SOD2, and negative controls included omitting the primary antibody and reacting sections with diluent buffer only and the use of an irrelevant rabbit antibody ( $\operatorname{IgG}$ isotype) with each staining set. Staining was evaluated using a semi-quantitive method as follows: -, negative; \pm , equivocal; + , strongly positive. Pathological assessment and all immune reactions were evaluated in a blinded manner by independent researchers who had no knowledge of the outcome of therapy for individual patients.

\section{Results}

Radioresistance of CNE1 and CNE2. To determine the radioresistance in CNE1 and CNE2 cells, clonogenic survival was determined, and the DMFs at $10 \%$ isosurvival were found to be 1.529 and 1 for CNE1 and CNE2 cells (relative to CNE2, Fig. 1A). Survival curve data were analyzed using a modified linear quadratic model the programme DRFIT. CNE1 cells demonstrated increased radioresistance as shown by a significant decrease in $\alpha$, increase in MID and increase in SF2, compare to CNE2 cells (Fig. 1A, right schematic diagram). There was no significant difference in the $\beta$ value. 
These results are in agreement with previous reports of celular radiobiological characteristics human NPC cell lines (25).

Increased SOD2 in relatively radioresistant CNE1 cells. Fig. 1 shows that, compared to CNE2 cells (Fig. 1B, line 2), levels of SOD2 expression were increased in CNE1 cells (lane 1). The SOD2 protein levels were estimated by densitometry and normalized to B-actin (Fig. 1B, lower panel), CNE1 cells were 1.94-fold higher than CNE2 cells at SOD2 protein base line and radioresistance correlated to SOD2 expression levels. Enzyme activity detected by water-soluble tetrazolium salt microplate assay further showed that endogenous SOD2 activity was increased in CNE1 cells.

Endogenous SOD2 expression in CNE1 and CNE2 cells exposed to IR. By using the SOD2/ß-actin ratio (Fig. 1C) series doses or time of radiation $1,2,4$ Gy or $6,12,24 \mathrm{~h}$ induced 1.15-fold to 1.39-fold increase in the level of SOD2 transcript in CNE1 cells, but not in CNE2 cells that constitutively demonstrated a 2-fold-greater level of endogenous SOD2 (Fig. 1C). These results indicate that the induction of SOD2 by radiation is more pronounced in CNE1 cells, relative to CNE2 cells.

Expression of miRNA for SOD2 sensitized CNE1 cells to IR. To determine if a causal relationship between SOD2 expression and radiosensitivity exists, CNE1 cells were transfected with the miRNA expression construct for RNAi targeting SOD2 (Fig. 2A). CNE1 cells stably transfected with pcDNATM6.2-GW/EmGFP-miR-SOD2 down-regulated the SOD2 mRNA levels and exhibited decreases in SOD2 immunoreactive protein (Fig. 2B). Cells from the parental line and those transfected with pcDNATM6.2-GW/EmGFPmiR-neg or pcDNATM6.2-GW/EmGFP-miR-SOD2 were irradiated with dose ranging from $0-8 \mathrm{~Gy}$, clonogenic survival was determined. The results (Fig. 2C) show that expression of miRNA for SOD2 inhibited resistance to radiation-induced cell kill, as shown by DMFs of 0.435 and 1.043 for CNE1+miR-SOD2 and CNE1+miR-neg cells, respectively, at $10 \%$ isosurvival (relative to CNE1). Survival curve data were analyzed using a modified linear quadratic model the programme DRFIT. CNE1 cells transduced with miR-SOD2 demonstrated decreased radioresistance as shown by a significant increase in $\alpha$, decrease in MID and decrease in SF2 (Fig. 2C, right schematic diagram). There was no significant difference in the $\beta$ value. Fig. $2 \mathrm{~B}$ shows reduced expression of SOD2 mRNA and protein in CNE1+miRSOD2 cell line. These observations support the hypothesis that SOD2 expression regulates a portion of the radioresistant phenotype.

Increased expression of SOD2 in radioresistant NPC tissues. The following variables, staging of tumor, sex and age, and treatment-planning method were not significantly different in the radioresistant tumor group and the radiosensitive tumor group. SOD2 expression was cytoplasmic. Heterogeneity was observed both in the percentage of cells positive and in the level of expression of protein: $11 / 23(47.8 \%)$ radioresistant tumors were SOD2-positive and 12/23 (52.2\%) were

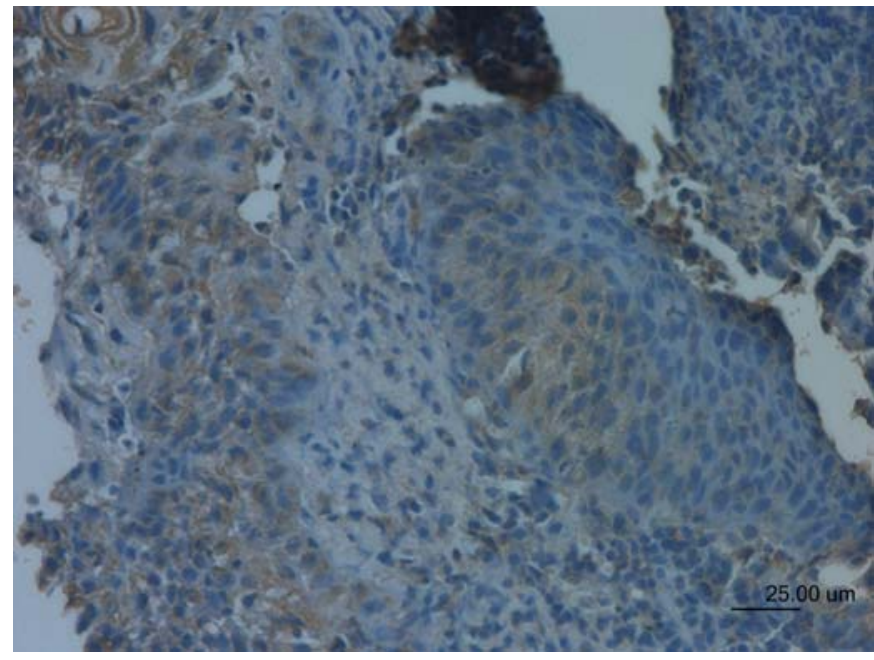

Figure 3. Immunohistochemical staining of SOD2 was performed on sections using the streptavidin-biotin peroxidase method. Tissue sections were deparaffinized and rehydrated in graded alcohols. Endogenous peroxide activity was quenched with methanol in hydrogen peroxide. Incubation in blocking serum was carried out prior to the application of primary antibody. A rabbit polyclonal antibody to SOD2 was used. The secondary antibody was mouse anti-rabbit IgG. The reaction was developed using an avidinbiotin-peroxidase complex. The chromagen 3,3-diaminobenzidine was used to identify sites of immunostain. For a lesion to be considered positive, it had to have intense immunostain in at least five tumor nuclei in a single high-power field.

Table I. Expression of SOD2 protein was monitored in radioresistant tumors and radiosensitive tumors.

\begin{tabular}{lccc}
\hline & Positive & Negative & $\begin{array}{c}\text { Positive } \\
\text { proportion (\%) }\end{array}$ \\
\hline $\begin{array}{l}\text { Radioresistant } \\
\text { group }\end{array}$ & 11 & 12 & 47.5 \\
$\begin{array}{l}\text { Radiosensitive } \\
\text { group }\end{array}$ & 2 & 44 & 4.5 \\
\hline
\end{tabular}

The proportion of SOD2-positive samples was compared between two groups using Fisher's exact test, $\mathrm{p}<0.01$.

SOD2-negative; $2 / 46(4.5 \%)$ radiosensitive tumors were SOD2-positive and 44/46 (95.5\%) were SOD2-negative before commencement of radiotherapy. Associations of expression of SOD2 protein with patient and tumor characteristics for radioresistance are shown in Table I.

\section{Discussion}

Carcinoma of the nasopharynx is prevalent in the South China region (26). The most effective means of treatment of NPC is generally radiation therapy $(27,28)$. Patients with NPC often have to face deleterious treatment consequences as the price of cure (29); the therapeutic ratio of routine fractionated radiation, however, has not improved over several decades and there is plenty of room for improvement of the treatment of NPC in China (30). The 5-year survival rate of 
nasopharyngeal carcinomas is about $50 \%$ overall (31) and failure to control the cancer is mainly due to a portion of radioresistant phenotype of NPC. Hence, therapeutic efficacy can be improved by devising an approach that will increase the response of the tumor relative to that of the surrounding normal tissues (32). The approach involves exploiting biological factors that result in differences in the response of tumors and normal tissues to radiation therapy. Thanks to the advancements in molecular medicine, specific treatment to the potential target using technologies such as immunotherapy and RNAi becomes a potential genetic marker of radiosensitivity discovery more meaningful for NPC management.

The mechanisms causing resistance to radiotherapy in NPC are poorly understood. However, identifying gene targets for radiosensitization is an important strategy in improving anticancer treatments (33-35). Exposure of cells to ionizing radiation leads to the formation of reactive oxygen species that are associated with radiation-induced cytotoxicity. The antioxidant enzyme manganese superoxide dismutase (SOD2) catalyzes the dismutation of the superoxide anions into hydrogen peroxide, to enhance the radioresistance of a mammalian cell. A number of studies in other body sites have shown cellular resistance to radiation-induced DNAdamaging in the overexpression of SOD2 (36). But the results of some studies are contradictory $(7,15)$. SOD2 may alter radioresistance via gene regulation. However, SOD2-mediated induction of radioresistance may be species and cell linedependent (8).

The present study provides the first evidence that SOD2 expression regulates a portion of the radioresistant phenotype in NPC cell lines. Indirect evidence in support of this conclusion includes the fact that CNE1 with relatively increased expression of SOD2 showed significant radioresistance compare to CNE2 with relatively decreased SOD2 expression, and endogenous SOD2 was induced in irradiated CNE1 cells. Further support was obtained when expression of SOD2 protein induced in CNE1 cells was also found to be reduced in CNE2 cells with low SOD2 protein base line following irradiation. A causal relationship between SOD2 expression and increased radiation resistance in NPC cell line was further suggested by experiments showing that expression of miRNA for RNA interference with SOD2 down-regulated SOD2 and significantly radiosensitized CNE1 cells. We have shown that enforced expression of miRNA for SOD2 in CNE1 cells significantly decreases radioresistance. SOD2 was found to be up-regulated in CNE1 cells, and the protein level was down-regulated following expression of miRNA for SOD2 in CNE1 cells during the same time that radiosensitization was occurring (Figs. 1 and 2). Therefore, we hypothesize that SOD2 may play a specific role in radioresistance relevant to nasopharyngeal carcinoma cell lines. Finally, in the study of 69 nasopharyngeal carcinomas, we have demonstrated that incidence of SOD2 immunostain in the radioresistant group was significantly higher than in the radiosensitive group. Expression of SOD2 protein may contribute to the radioresistance of some nasopharyngeal carcinomas. The present results provide evidence for SOD2 expression participating in radioresistance.

Although the mechanism by which SOD2 impacts resistance to ionizing radiation is currently unknown, SOD2 overexpression has been suggested by other studies to alter the intracellular redox environment via changes in mitochondial hydroperoxide production (37), initiating downstream signaling cascades and transcription factors that control the stress-responsive signaling pathways (38). The stress-signaling proteins responsive to SOD2 expression have been correlated to the signaling pathways governing radiation response and radioresistance. Down-regulated cyclin B1 increases radiosensitization (39); 14-3-3 proteins are phosphoserine-binding molecules with a primary function of inhibiting apoptosis (40); c-Rel controled SOD2 was found to play a key role in antiapoptosis (41); E2F1 and c-Myc appear to potentiate apoptosis through inhibition of NF- $\mathrm{B}$ activity that facilitates SOD2-mediated ROS elimination (42). A pathway activated by SOD2 to effector genes with antiapoptotic function is a contributor to radiation-induced adaptive responses (8) that results in enhanced tolerance to the subsequent cytotoxicity of ionizing radiation. In addition, modifying intracellar or extracellar SOD activity reduce the oxygen enhancement ratio leading to the effects of oxygen on radiosensitization (1). SOD2 with superoxide dismutation capability following radiation could result in radioprotection.

Our study suggests that the levels of SOD2 expression, which can be rapidly applied, are important markers of a subset of tumors (and thus of patients) in which fractionated radiation treatment failure is likely. Further work is clearly required for a complete understanding of what extent of SOD2 expression are responsible. In this regard, several authors have recently reported that overexpression of SOD2 may result in protecting normal tissues from radiation injury $(6,14,18)$, and endogenous SOD activity is also involved in protecting human leukemic and cancer cells from radiation (21), confirming that detailed analysis of SOD2 gene expression and their biological functions should be considered (43). If future studies confirm that SOD2 expression are associated with a tumor residual or confer a radioresistance when a particular radiation treatment-planning method is used, then identifying patients with tumors that carry increased SOD2 may improve treatment selection. Furthermore, regulating the expression of SOD2 in vitro and in mouse model has improved the sensitization of cell lines and experimental tumors to radiation therapy, or the protection of the haematopoietic compartment and normal tissues from the effects of ionizing radiation, demonstrating the effectiveness of a combination of gene therapy and conventional radiotherapy $(10,15,44-46)$. Accurate detection of SOD2 status prior to therapy will also allow SOD2 inhibitors or radiosensitizers to be used in clinical trials in NPC to reduce failure from radiotherapy. Our findings therefore have important therapeutic implications for the future in moving towards an improved cancer therapy for NPC.

\section{Acknowledgements}

We thank Dr Xinzhang Cai for insightful advice and Mr. Chenglong Wang for technical assistance.

\section{References}

1. Oberley LW, Lindgren AL, Beker SA, et al: Superoxide ion as the cause of the oxygen effect. Radiat Res 68: 320-328, 1976.

2. Biaglow JE, Mitchell JB and Held K: The importance of peroxide and superoxide in the X-ray response. Int J Radiat Oncol Biol Phys 22: 665-669, 1992. 
3. Hall EJ: Radiobiology for the radiologist. Lippincott Williams and Wilkins, Philadelphia, 2000.

4. Wispe JR, Clark JC, Burhans MS, et al: Synthesis and processing of the precursor for human mangano-superoxide dismutase. Biochim Biophys Acta 994: 30-36, 1989.

5. Gajewska J, Szczypka M, Izbicki T, et al: Antioxidant and glutathione-associated enzymes in Wilms' tumour after chemotherapy. J Cancer Res Clin Oncol 122: 483-488, 1996.

6. Epperly MW, Epstein CJ, Travis EL, et al: Decreased pulmonary radiation resistance of manganese superoxide dismutase (MnSOD)-deficient mice is corrected by human manganese superoxide dismutase-plasmid/Liposome (SOD2-PL) intratracheal gene therapy. Radiat Res 154: 365-374, 2000.

7. Vujaskovic Z, Batinic-Haberle I, Rabbani ZN, et al: A small molecular weight catalytic metalloporphyrin antioxidant with superoxide dismutase (SOD) mimetic properties protects lungs from radiation-induced injury. Free Radic Biol Med 33: 857-863, 2002.

8. Guo GZ, Yan-Sander Y, Lyn-Cook BD, et al: Manganese superoxide dismutase-mediated gene expression in radiationinduced adaptive responses. Mol Cell Biol 23: 2362-2378, 2003

9. Lefaix JL, Delanian S, Leplat JJ, et al: Successful treatment of radiation-induced fibrosis using $\mathrm{Cu} / \mathrm{Zn}-\mathrm{SOD}$ and MnSOD: an experimental study. Int J Radiat Oncol Biol Phys 35: 305-312, 1996.

10. Greenberger JS, Epperly MW, Gretton J, et al: Radioprotective gene therapy. Curr Gene Ther 3: 183-195, 2003.

11. Kang SK, Rabbani ZN, Folz RJ, et al: Overexpression of extracellular superoxide dismutase protects mice from radiationinduced lung injury. Int J Radiat Oncol Biol Phys 57: 1056-1066, 2003

12. Azzam EI, Black SM, Schmidt-Ullrich RK, et al: Oxidative metabolism modulates signal transduction and micronucleus formation in bystander cells from $\alpha$-particle-irradiated normal human fibroblasts. Cancer Res 62: 5436-5442, 2002.

13. Oberley LW, St. Clair DK, Autor AP, et al: Increase in manganese superoxide dismutase activity in the mouse heart after X-irradiation. Arch Biochem Biophys 254: 69-80, 1987.

14. Summers RW, Maves BV, Reeves RD, et al: Irradiation increase superoxide dismutase in rat intestinal smooth muscle. Free Radic Biol Med 6: 261-270, 1989.

15. Epperly MW, DeFilippi S, Sikora CA, et al: Intratrachea injection of manganese superoxide dismutase (MnSOD) plasmid/ liposomes protects normal lung but not orthotopic tumors from irradiation. Gene Ther 7: 1011-1018, 2000.

16. Li JJ, Oberley LW, St. Clair D, et al: Phenotypic changes induced in human breast cancer cells by overexpression of manganese-containing superoxide dismutase cDNA. Oncogene 10: 1989-2000, 1995.

17. Oberley LW: Anticancer therapy by overexpression of superoxide dismutase. Antioxid Redox Signal 3: 461-472, 2001.

18. Yan T, Oberley LW, Zhang W, et al: Manganese-containing superoxide dismutase overexpression cause phenotypic reversion in SV-40 transformed human lung fibroblasts. Cancer Res 56: 1864-1871, 1996.

19. Zhong W, Oberley LW, Oberley TD, et al: Inhibition of cell growth and sensitization to oxidative damage by overexpression of manganese superoxide dismutase in rat glioma cells. Cell Growth Differ 7: 1175-1186, 1996.

20. Ioannides C and Lewis DFC: Drugs, diet and disease. Ellis Horwoord, Hemel Hemstead, pp47-63, 1994.

21. Yamaguchi S, Sakurada S and Nagumo M: Role of intracellular SOD in protecting human leukemic and cancer cells superoxide and radiation. Free Radic Biol Med 17: 389-395, 1994.

22. Asano S, Imano M, Kuroda D, et al: Expression of manganese superoxide dismutase in esophageal and gastric cancers. J Gastroenterol 33: 816-822, 1998.

23. Veldwijk MR, Herskind C, Laufs S, et al: Recombinant adenoassociated virus 2-mediated transfer of the human superoxidedismutase gene does not confer radioresistance on HeLa cervical carcinoma cells. Radiother Oncol 72: 341-350, 2004.
24. Roberts SA: DRFIT: a program for fitting radiation survival models. Int J Radiat Biol 57: 1243-1246, 1990.

25. Xia YF, Li MZ, Huang B, et al: Celular radiobiological characteristics of human nasopharyngeal carcinoma cell lines Chin J Cancer 20: 683-687, 2001

26. Parkin DM, Muir CS, Whelan SL, et al: Cancer incidence in five continents. Vol 6. IARC Science Publications, Lyon, p120, 1992.

27. Wang CC: Treatment of malignant tumours of the nasopharynx. Otolaryngol Clin North Am 13: 477-481, 1980.

28. Ho JHC: Nasopharynx. In: Treatment of Cancer. Halnan KE, Boak JL, Crowther D, von Essen CF, Orr JS and Peckham MJ (eds). Chapman and Hall, pp249-267, 1982.

29. Marks JE, Bedwinek JM, Lee F, et al: Dose-response analysis for nasopharyndeal carcinoma: an historical perspective. Cancer 50: $1042-1050,1982$

30. Zhang YW: Progress on diagnosis and therapy for nasopharyngeal carcinoma. In: New Theories and Technologies in Oncology (In Chinese). Cao SL (ed). Scientific and Technical Publishers, Shanghai, p700, 1997.

31. Greene FL, Page DL, Fleming ID, et al: AJCC Cancer Staging Manual. 6th edition. Springer, New York, p38, 2002.

32. Tannock IF and Hill RP: The basic science of oncology. 3rd edition. McGraw-Hill, Singapore, pp219-237, 1999.

33. Coleman CN and Stevenson MA: Biologic basis for radiation oncology. Oncology 10: 399-411, 1996.

34. Hahn SM, Russo A, Cook JA, et al: A multidrug-resistant breast cancer cell line induced by weekly exposure to doxorubicin. Int J Oncol 14: 273-279, 1999.

35. Mitchell JB, Russo A, Cook JA, et al: Tumor cell drug and radiation resistance: does an interrelationship exist? Cancer Treat Res 48: 189-203, 1989.

36. Epperly MW, Sikora CA, DeFilippi SJ, et al: Manganese superoxide dismutase (SOD2) inhibits radiation-induced apoptosis by stabilization of the mitochondrial membrance. Radiat Res 157: 568-577, 2002.

37. Li S, Yan T, Yang JQ, et al: The role of cellular glutathione peroxdase redox regulation in the suppression of tumor cell growth by manganese superoxide dismutase. Cancer Res 60: 3927-3939, 2000

38. Zhang HJ, Zhao W, Venkataraman S, et al: Activation of matrix metalloproteinase-2 by overexpression of manganese superoxide dismutase in human breast cancer MCF-7 cells involves reactive oxygen species. J Biol Chem 277: 20919-20926, 2002.

39. Li Z, Xia L, Lee ML, et al: Effector genes altered in MCF-7 human breast cancer cells after exposure to fractionated ionizing radiation. Radiat Res 155: 543-553, 2001.

40. Xing H, Zhang S, Weinheimer C, et al: 14-3-3 Proteins block apoptosis and differentially regulate MAPK cascades. EMBO J 19: 349-358, 2000

41. Bernard D, Monte D, Vandenbunder B, et al: The c-Rel transcription factor can both induce and inhibit apoptosis in the same cells via the up-regulation of MnSOD. Oncogene 21: 4392-4402. 2002.

42. Tanaka H, Matsumura I, Ezoe S, et al: E2F1 and c-Myc potentiate apoptosis through inhibition of NF-kappaB activity that facilitates MnSOD-mediated ROS elimination. Mol Cell 9: 1017-1029, 2002

43. Spitz DR, Azzam EI, Li JJ, et al: Metabolic oxidation/reduction reactions and cellular responses to ionizing radiation: a unifying concept in stress response biology. Cancer Met Rev 23: 311-322, 2004.

44. Southgate TD, Sheard V, Milsom MD, et al: Radioprotective gene therapy through retroviral expression of manganese superoxide dismutase. J Gene Med 8: 557-565, 2006.

45. Guo HL, Wolfe D, Epperly MW, et al: Gene transfer of human manganese superoxide dismutase protects small intestinal villi from radiation injury. J Gastrointest Surg 7: 229-236, 2003.

46. Kanai AJ, Zeidel ML, Lavelle JP, et al: Manganese superoxide dismutase gene therapy protects against irradiation-induced cystitis. Am J Physiol Renal Physiol 283: F1304-F1312, 2002. 